\title{
KS Inertial Range and Validity of Richardson Regime
}

\author{
F. Nicolleau* and A. Abou El-Azm Aly ${ }^{* *}$
}

\begin{abstract}
It has been argued in [10] that due to the lack of sweeping of small scales by large scales in kinematic simulation, the validity of the Richardson's power law might be affected. Here, we argue that the discrepancies between the authors on the ability of kinematic simulation, KS, to predict Richardson power law may be linked to the inertial subrange they have used. For small inertial subrange, KS is efficient and the significance of the sweeping can be ignored, as a result we limit the KS agreement with the Richardson scaling law $\mathrm{t}^{3}$ for inertial subrange $\mathrm{k}_{\mathrm{N}} / \mathrm{k}_{1} \leq 10000$. Above this value, the sweeping effect of the small scales by the large scales must be taken in considerations where KS failed to reproduces this power law in that range as obtained in [13].
\end{abstract}

Keywords: Richardson Regime, kinematic simulation, two-particle separation.

\section{Introduction}

Two-particle separation is defined as:

$\Delta(\mathrm{t})=\mathrm{X}_{2}(\mathrm{t})-\mathrm{X}_{1}(\mathrm{t})$

where $X_{1}(t)$ is the position of the first particle and $X_{2}(t)$ the position of the second particle at time $\mathrm{t}$.

Two-particle relative separation is defined as:

$\Delta_{\mathrm{r}}(\mathrm{t})=\left|\left(\mathrm{X}_{2}(\mathrm{t})-\mathrm{X}_{2}\left(\mathrm{t}_{0}\right)\right)-\left(\mathrm{X}_{1}(\mathrm{t})-\mathrm{X}_{1}\left(\mathrm{t}_{0}\right)\right)\right|$

where $t_{0}$ is the time of release of the particles or initial time. Two-particle separation and relative separation have received much research attention since the pioneering work of [1]. This separation depends on the concentration covariance and not on the average concentration. Consequently, this concentration covariance is governed by some features of two-particle statistics [2]. To characterize these statistics of two-particle separation, the mean-square separation between the two particles $\mathrm{X}_{1}(\mathrm{t})$ and $\mathrm{X}_{2}(\mathrm{t}),<\Delta^{2}(\mathrm{t})>$ has been used, where $\Delta(\mathrm{t})=\mathrm{X}_{2}(\mathrm{t})-\mathrm{X}_{1}(\mathrm{t})$. This is the first quantity to understand in the study of the twoparticle separation in turbulent flow.

\footnotetext{
${ }^{*}$ Senior Lecturer, Department of Mechanical Engineering, The University of Sheffield, UK.

Egyptian Armed Forces.
} 
Richardson [1] studied the diffusivity $\mathrm{d} / \mathrm{dt}\left\langle\Delta^{2}(\mathrm{t})\right\rangle$ as a function of the mean-square separation, $\left\langle\Delta^{2}(\mathrm{t})>\right.$, between two particles in the inertial subrange of turbulence. That is when $\eta<\Delta(\mathrm{t})<\mathrm{L}$, where $\mathrm{L}$ is the upper limit length scale of the inertial range, Richardson introduced the locality assumption and derived his four-third law of diffusion:

$$
\mathrm{d} / \mathrm{dt}<\Delta^{2}(\mathrm{t})>\approx \Delta^{4 / 3}(\mathrm{t})
$$

His locality assumption states that the mean square separation reaches a limit as the averaging time is increased because only eddies comparable in size with the separation are effective. Neglecting the initial separations $\Delta_{0}, \varepsilon$ is the rate of dissipation energy per unit mass and applying Kolmogorov's similarity theory to the relative diffusion of particles, Obukhov [3] and Batchelor [4] obtained the famous $\mathrm{t}^{3}$ law for diffusion in isotropic turbulence.

$<\Delta^{2}(\mathrm{t})>=\mathrm{G}_{\Delta} \varepsilon \mathrm{t}^{3}$

where $\mathrm{G}_{\Delta}$ is the Richardson universal dimensionless constant in an intermediate inertial range of times and of order of 0.1. This latter equation implies Richardson's diffusivity law (3) and therefore suggests that his locality assumption applies to the two-particle diffusion in the inertial range.

From that time, many researches have been carried out both to validate this power scaling and to validate numerical methods on that power law. Validation of the power law (4) have been attempted either experimentally as in [5] in a two-dimensional turbulent flow but to a limiting Reynolds number or numerically as in [6]. They have been faced with a limiting Reynolds number not large enough to get clear inertial subrange statistics.

Here we study the case of Kinematic Simulation which allows one to reach a high enough Reynolds number to get a reasonable inertial subrange statistics to validate the Richardson's power law. Many studies have been done using KS to study the turbulent diffusion of particle pairs. This has been done either to validate the power law in different ranges of Reynolds numbers or to find a specified value for the Richardson's constant, $G_{\Delta}$, which still has uncertainties in its value. There has been some contradictory conclusion as to the ability of $\mathrm{KS}$ to predict a $\mathrm{t}^{3}$ law. According to [2, 7-9] KS predict (3), according to [10] they do not.

To clarify the situation, we analyze, in each study, the inertial subrange which is used to study the particle diffusion. Fung and Vassilicos [2] investigated two-particle diffusion in a twodimensional turbulent flow using KS and study the locality assumption, the fractal-eddy structure and the straining regions' role in separating the particle pairs. They validated the locality scaling for power spectrum exponent $\leq 3$ spanning more than one decade, with an inertial subrange $\mathrm{k}_{\mathrm{N}} / \mathrm{k}_{1}=4000$. Moreover, in [7], Malik and Vassilicos made the first direct comparison between the results obtained from Yeung's direct numerical simulation [6] and those obtained from KS. This reflects the first validation test for KS as a serious Lagrangian modeling tool for fluid particle pair diffusion in an isotropic, three dimensional turbulent flows. The simulated inertial subrange was in the range $\mathrm{k}_{\mathrm{N}} / \mathrm{k}_{1}=400$. They concluded that their results were in agreement with those obtained by DNS. They also showed that the unsteadiness parameter that is used as an input in KS has no significant effect on the Lagrangian statistics.

Later in [11], Nicolleau and Vassilicos used the kinematic simulation technique to model particle pair separations in a two-dimensional turbulent flow. Their results were obtained for 
an inertial subrange up to $\mathrm{k}_{\mathrm{N}} / \mathrm{k}_{1}=250000$. They succeeded to reproduce the laboratory results of [5]. However, they could not observe the Richardson regime at high inertial subrange, $\mathrm{k}_{\mathrm{N}} / \mathrm{k}_{1}=38748$, except at certain initial separations, $\Delta_{0}=\eta$ and $\Delta_{0}=0.1 \eta$. Their deviation from the Richardson's law without the dependency of the initial separations was left as an open question. In [8], Davila and Vassilicos used KS to determine the relation between Richardson's power law exponent for the pair separation, $\gamma$, and the fractal dimension of the stagnation points, $D_{s}$, in isotropic turbulent flow. They found this relation to be $\gamma=6 / D_{s}$ for the inertial subrange $\mathrm{k}_{\mathrm{N}} / \mathrm{k}_{1}=1000$ for different values of the energy spectrum exponent $1 \leq \mathrm{p}$ $\leq 2$. Moreover, they found that the Richardson constant is an increasing function of $D_{s}$. In [9], Nicolleau and $\mathrm{Yu}$ studied two-particle diffusion in a three-dimensional statistically isotropic turbulent flow using KS for different power law exponent of the energy spectrum from 1.2 to 3 and its effect on two-particle diffusion. But they limit this study to $\mathrm{k}_{\mathrm{N}} / \mathrm{k}_{1}=$ 2000. They validated this range over a range of scales and also studied two-particle mean diffusivity when the two-particle separation is larger than Kolmogorov length scale.

Recently in [10], Thomson and Devenish have investigated the particle pair separation using KS with a particular attention paid to the problems caused by the lack of sweeping of small scales by the larger scales in KS. As a consequence, they expected that the mean-square separation would grow in a different way from Richardson scaling because the large scales can influence the rate of separation. In the case of flows with a large mean velocity, the scaling grows as $\mathrm{t}^{6}$ and in the case where there is no mean velocity the scaling grows as $\mathrm{t}^{9 / 2}$. This was confirmed by KS numerical simulations for an inertial subrange range $k_{N} / k_{1}=10^{6}$ and $10^{8}$ which conflicts with Richardson's power law in real flows. Finally they raised a question about the applicability of KS to produce the particle pair statistics as in real flow.

As a reply, in [12], Osborne et al have investigated the separation of particle pairs using kinematic simulation with attention to the argument that has been raised by [10] for the problem caused by the lack of sweeping of the small scales by the large scales found in KS. They simulated the inertial subrange in the range $\mathrm{k}_{\mathrm{N}} / \mathrm{k}_{1}=10^{4}$. They have argued that the main difference between their results and those obtained in [10] is due to the integration time step where they used a constant time step lower than the lowest time scale while in [10]. They have used an adaptive time step depending on the instantaneous separation of the particles. They concluded that, for the results predicting the temporal scaling of fluid pair separation based on the lack of the KS to the sweeping of small scales by large scales, KS reproduces Richardson's power law over a wide range of scales and not as the one predicted in $[10]$.

Consequently in [13], Devenish and Thomson changed the adapted time step used in their previous results [10] to small fixed time step. They studied the particle pair separation using KS with the same attention paid to the resulting mean-square separation to obey Richardson' scaling $\mathrm{t}^{3}$ in turbulent flow with the same inertial subrange range $\mathrm{k}_{\mathrm{N}} / \mathrm{k}_{1}=10^{6}$ and $10^{8}$. They confirmed that with the new time step used they got the same trend of the mean-square separation to grow as $\mathrm{t}^{6}$ in the case of large mean flow and as $\mathrm{t}^{9 / 2}$ in the case of no mean flow. They concluded with these numerical simulations that KS is unable to represent the separation scaling as in real flow. This was consistent with their previous results in [10] because of the lack of sweeping of small scales by the larger scales in KS.

In the present work, we study particle pair separations in an isotropic turbulent flow using KS and investigate the ability of this method to reproduce the well known Richardson's law. The 
numerical approach used to generate turbulent flow field is introduced in section 2, the results obtained are presented in section 3 .

\section{Kinematic simulation technique}

Kinematic Simulation is a Lagrangian model for turbulent diffusion based on a simplified incompressible velocity field which kinematically simulates the Eulerian velocity field and is generated as a sum of random incompressible Fourier modes with a proper wave number energy spectrum and only modeled the inertial subrange of the energy spectrum. The interactions between the random Fourier modes are not modeled hence KS miss their dynamics which is called the lack of sweeping between different modes, this fact has been argued in [12] for one-particle statistics and argued for two-particle statistics as in [10] and recently in [13].

The computational simplicity of $\mathrm{KS}$ allows one to consider large inertial sub-ranges and Reynolds numbers, Re. With this method, the computational task reduces to the calculation of the trajectory of each particle placed in the turbulent field; each trajectory is, for a given initial condition, solution of the differential equation:

$\mathrm{dx} / \mathrm{dt}=\mathbf{u}_{\mathrm{E}}(\mathrm{x}, \mathrm{t})$

where $\mathbf{u}_{\mathrm{E}}$ is the Eulerian velocity supposed to be given by KS. This is done by integrating Equation (5) using the $4^{\text {th }}$ order predictor corrector method (Adams-Bashforth-Moulton) in which Runge-Kutta-4 is used to compute the first three points in the Adams-Bashforth's method. For each realization of the velocity field, the trajectories for each particle have been computed simultaneously. This kind of computation does not require the storage of a lot of data with very big tables as with direct numerical simulation. As in $[2,14]$, the 3-D KS turbulent velocity field in this paper is kinematically presented by a truncated Fourier series, sum of $\mathrm{N}$ random Fourier modes:

$u_{E}(x, t)=\sum_{n=1}^{N_{k}}\left[\left(a_{n} \times \hat{k}_{n}\right) \cos \left(k_{n} \cdot x+\omega_{n} t\right)+\left(b_{n} \times \hat{k}_{n}\right) \sin \left(k_{n} \cdot x+\omega_{n} t\right)\right]$

where $\mathrm{N}$ is the total number of Fourier modes, the wave vector $\mathbf{k}_{\mathrm{n}}$ is randomly oriented. The vectors $\mathbf{a}_{\mathrm{n}}$ and $\mathbf{b}_{\mathrm{n}}$ are chosen independently and randomly in a plane normal to $\mathbf{k}_{\mathrm{n}}$ to ensure that the velocity field is incompressible $\nabla . \mathbf{u}=0$. Their amplitudes have been chosen to be consentient with the prescribed energy spectrum.

The wave number distribution is chosen as follows:

$\mathrm{k}_{\mathrm{n}}=\mathrm{k}_{1}\left(\mathrm{k}_{\mathrm{N}} / \mathrm{k}_{1}\right)((\mathrm{n}-1) /(\mathrm{N}-1))$

The Kolmogorov length scale is defined as $\eta=2 \pi / \mathrm{k}_{\mathrm{N}}$. The ratio between the integral and Kolmogorov length scales is $\mathrm{L} / \eta=\mathrm{k}_{\mathrm{N}} / \mathrm{k}_{1}$ which is used to determine the inertial range and the associated Reynolds number: $\operatorname{Re}=(\mathrm{L} / \eta)^{4 / 3}=\left(\mathrm{k}_{\mathrm{N}} / \mathrm{k}_{1}\right)^{4 / 3}$. In this study, we will use an energy spectrum characterized by a power law with an exponent, $\mathrm{p}$, varying from 1.27 to 1.97: 


$$
E(k)=\left\{\begin{array}{cc}
C_{k} \varepsilon^{2 / 3} k^{-p} & k_{l}<k<k_{N} \\
0 & \text { otherwise }
\end{array}\right.
$$

where $\mathrm{C}_{\mathrm{k}}$ is the Kolmogorov constant $\left(\mathrm{C}_{\mathrm{k}}=1.5\right)$ [15] and $\varepsilon$ is the dissipation rate of energy per unit mass $\left(\varepsilon=u^{3} / \mathrm{L}\right)$. The rms of the turbulent velocity fluctuation is:

$$
u^{\prime}=\sqrt{\frac{2}{3} \int_{k_{1}}^{k_{N}} E(k) d k}
$$

The integral length scale of the isotropic turbulence is defined as follows:

$L=\frac{3 \pi}{4} \frac{\int_{k_{1}}^{k_{N}} k^{-1} E(k) d k}{\int_{k_{1}}^{k_{N}} E(k) d k}$

The unsteadiness frequency, $\omega_{\mathrm{n}}$, determines the unsteadiness associated with the $\mathrm{n}^{\text {th }}$ wave mode and is proportional to the eddy turnover frequency of that mode as:

$\omega_{\mathrm{n}}=\lambda\left(\mathrm{k}_{\mathrm{n}}^{3} \mathrm{E}\left(\mathrm{k}_{\mathrm{n}}\right)\right)^{1 / 2}$

where $\lambda$ is the unsteadiness parameter, this parameter enables one to create three-dimensional effects in the case of a two-dimensional simulation.

The value of the first wave number, $\mathrm{k}_{1}$, has been set to $2 \pi$ for all simulations and the number of modes used has been set to $\mathrm{N}_{\mathrm{k}}=200$ for different inertial subranges. It has been shown [7] that in three-dimensional isotropic KS for two-particle diffusion, most of the statistical properties are insensitive to the unsteadiness parameter's value, provided that it rests in the range $0 \leq \lambda \leq 1$. In accordance with these results we have not added any unsteadiness term $(\lambda=0)$ in most of our KS simulations except to explain its effect on the validity of Richardson's regime. The turnover time is defined as $t_{d}=L / u$ ' and the Kolmogorov time scale is $t_{\eta}\left(\right.$ defined as $\left.t_{\eta}=t_{d}(\eta / L)^{2 / 3}\right)$.

\section{KS and Richardson Regime Validation}

The equation of motion, Equation (5), was integrated over 4000 realizations of the flow field. Runs have been made for $10^{3} \leq \mathrm{k}_{\mathrm{N}} / \mathrm{k}_{1} \leq 10^{8}$; in order to study the effect of the Reynolds number. We fix the initial separation to be $\Delta_{0} / \eta=10$ to make a direct comparison with the results obtained in [13]. All the runs parameters are tabulated in Table 1 for initial separation $\Delta_{0} / \eta=10$, r.m.s. velocity $\mathrm{u}^{\prime}=1 \mathrm{~ms}^{-1}$ and turnover time of the largest scale $\mathrm{t}_{\mathrm{d}}=1 \mathrm{~s}$.

Figure 1 shows the results obtained from KS compared with the results obtained from [13] at a high inertial subrange $\mathrm{k}_{\mathrm{N}} / \mathrm{k}_{1}=10^{8}$. It can be noticed that our KS model is able to reproduce approximately the same results that obtained by [13] with small deviations. Consequently, we will use this model to show that the validation of the Richardson regime using KS dependents on the length of the inertial subrange. 
Table 1 Different cases studied for two-particle separations

\begin{tabular}{|c|c|c|c|c|c|c|c|c|c|}
\hline Case & $\mathbf{k}_{\mathbf{N}} / \mathbf{k}_{\mathbf{1}}$ & $\mathbf{p}$ & $\boldsymbol{\lambda}$ & $\boldsymbol{\eta}$ & $\mathbf{C a s e}$ & $\mathbf{k}_{\mathbf{N}} / \mathbf{k}_{\mathbf{1}}$ & $\mathbf{p}$ & $\boldsymbol{\lambda}$ & $\boldsymbol{\eta}$ \\
\hline $\mathrm{A}$ & $10^{3}$ & 1.67 & 0 & $6.2810^{-3}$ & $\mathrm{~N}$ & $10^{5}$ & 1.67 & 0 & $10^{-5}$ \\
\hline $\mathrm{B}$ & $10^{4}$ & 1.27 & 0 & $6.2810^{-4}$ & $\mathrm{O}$ & $10^{6}$ & 1.27 & 0 & $10^{-6}$ \\
\hline $\mathrm{C}$ & $10^{4}$ & 1.37 & 0 & $6.2810^{-4}$ & $\mathrm{P}$ & $10^{6}$ & 1.37 & 0 & $10^{-6}$ \\
\hline $\mathrm{D}$ & $10^{4}$ & 1.47 & 0 & $6.2810^{-4}$ & $\mathrm{Q}$ & $10^{6}$ & 1.47 & 0 & $10^{-6}$ \\
\hline $\mathrm{E}$ & $10^{4}$ & 1.57 & 0 & $6.2810^{-4}$ & $\mathrm{R}$ & $10^{6}$ & 1.57 & 0 & $10^{-6}$ \\
\hline $\mathrm{F}$ & $10^{4}$ & 1.67 & 0 & $6.2810^{-4}$ & $\mathrm{~S}$ & $10^{6}$ & 1.67 & 0 & $10^{-6}$ \\
\hline $\mathrm{G}$ & $10^{4}$ & 1.67 & 0.25 & $6.2810^{-4}$ & $\mathrm{~T}$ & $10^{6}$ & 1.67 & 0.5 & $10^{-6}$ \\
\hline $\mathrm{H}$ & $10^{4}$ & 1.67 & 0.5 & $6.2810^{-4}$ & $\mathrm{U}$ & $10^{6}$ & 1.67 & 1 & $10^{-6}$ \\
\hline $\mathrm{I}$ & $10^{4}$ & 1.67 & 0.75 & $6.2810^{-4}$ & $\mathrm{~V}$ & $10^{6}$ & 1.77 & 0 & $10^{6}$ \\
\hline $\mathrm{J}$ & $10^{4}$ & 1.67 & 1 & $6.2810^{-4}$ & $\mathrm{~W}$ & $10^{6}$ & 1.87 & 0 & $10^{-6}$ \\
\hline $\mathrm{K}$ & $10^{4}$ & 1.77 & 0 & $6.2810^{-4}$ & $\mathrm{X}$ & $10^{6}$ & 1.97 & 0 & $10^{-6}$ \\
\hline $\mathrm{L}$ & $10^{4}$ & 1.87 & 0 & $6.2810^{-4}$ & $\mathrm{Y}$ & $10^{7}$ & 1.67 & 0 & $10^{-7}$ \\
\hline $\mathrm{M}$ & $10^{4}$ & 1.97 & 0 & $6.2810^{-4}$ & $\mathrm{Z}$ & $10^{8}$ & 1.67 & 0 & $10^{-8}$ \\
\hline
\end{tabular}

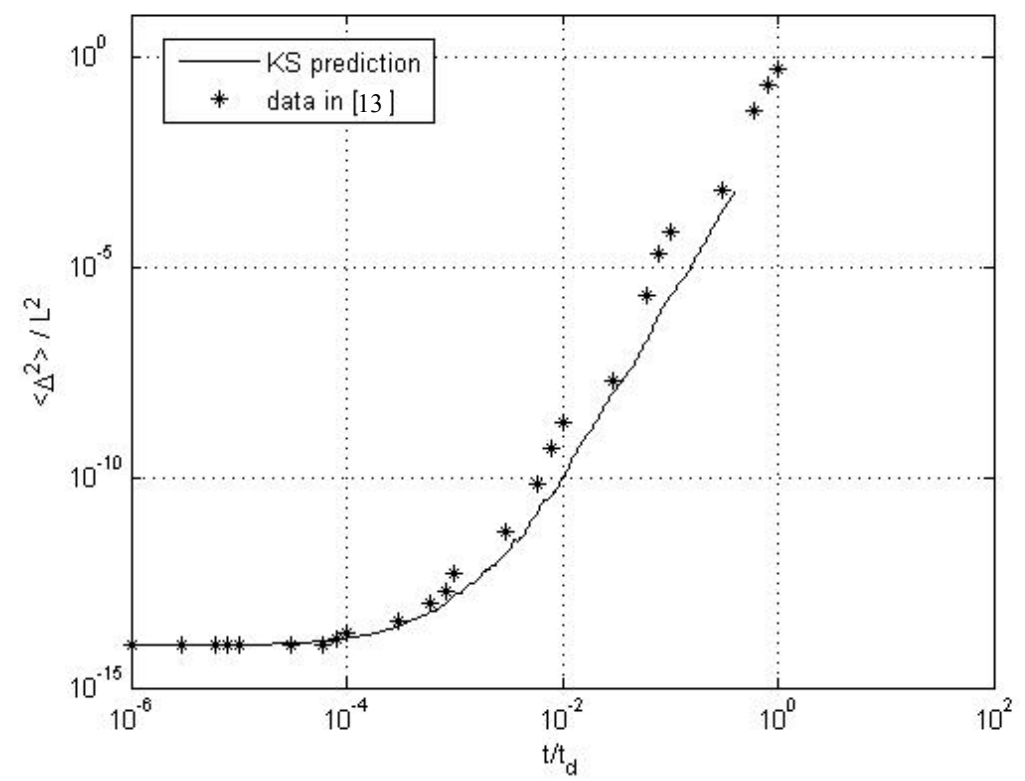

Fig. 1 Particle pair separation results obtained from KS; solid line from the current simulation, stars from the results obtained from [13], (case $\mathrm{Z}$ in Table 1)

\subsection{Particle pair diffusivity:}

In Figure 2, the particle pair separations $<\Delta^{2}>/ \mathrm{L}^{2}$ are plotted as a function of time for different inertial subranges, $10^{3} \leq \mathrm{k}_{\mathrm{N}} / \mathrm{k}_{1} \leq 10^{6}$. The slope of Richardson's scaling $\left(\mathrm{t}^{3}\right)$ and the slope proposed in [13] $\left(\mathrm{t}^{9 / 2}\right)$ are added to the figure.

It can be noticed that for small inertial subranges, up to $\mathrm{k}_{\mathrm{N}} / \mathrm{k}_{1}=10^{4}$, the curves seem to follow Richardson's scaling $\mathrm{t}^{3}$, but for higher inertial ranges they seem rather to follow the scaling $\mathrm{t}^{9 / 2}$. As mentioned in $\backslash$ the introduction, for [1] the reference quantity was the two-particle 
diffusivity. The locality in scale assumption was made for the diffusivity. Working directly on:

$(\mathrm{d} / \mathrm{dt})<\Delta^{2}(\mathrm{t})>\sim<\Delta^{2}(\mathrm{t})>^{2 / 3}$

is keeping closer to this fundamental assumption. [9] argued that plots of pair separations as functions of time as in Figure 2 can be misleading and their analysis need to be complemented by an analysis in terms of diffusivity.

In particular, conclusions are easier to draw from plots of $(\mathrm{d} / \mathrm{dt})<\Delta^{2}(\mathrm{t})>$ as they remove part of the initial separation $\Delta_{0}$ effects. Accordingly, in Figure 3 we plot $(\mathrm{d} / \mathrm{dt})\left\langle\Delta^{2}(\mathrm{t})\right\rangle /\left\langle\Delta^{2}(\mathrm{t})\right\rangle^{2 / 3}$ as a function of $<\Delta^{2}>/ \mathrm{L}^{2}$ for the different cases of Figure 2. A horizontal trend will validate each law respectively.

Whereas, for comparison, we plot $\left.(\mathrm{d} / \mathrm{dt})<\Delta^{2}(\mathrm{t})>/<\Delta^{2}(\mathrm{t})\right\rangle^{7 / 9}$ in Figure 4 as a function of $<\Delta^{2}>/ \mathrm{L}^{2}$ for the same cases.

Comparing both figures we can conclude that

$(\mathrm{d} / \mathrm{dt})<\Delta^{2}>\sim<\Delta^{2}>^{2 / 3}$

for inertial ranges $k_{N} / k_{1} \leq 10^{4}$. For larger ranges, Equation (13) is verified only after large separations, i.e. when $\Delta / \mathrm{L} \geq 10^{-2}$. Otherwise:

$(\mathrm{d} / \mathrm{dt})<\Delta^{2}>\sim<\Delta^{2}>^{7 / 9}$ for $\mathrm{k}_{\mathrm{N}} / \mathrm{k}_{1}>10^{4}$ and $\Delta / \mathrm{L}<10^{-2}$

In other words KS seems to struggle with Richardson's locality-in-scales hypothesis at small scales, not at large scales, in contrast to what was first suggested by the results in [10].

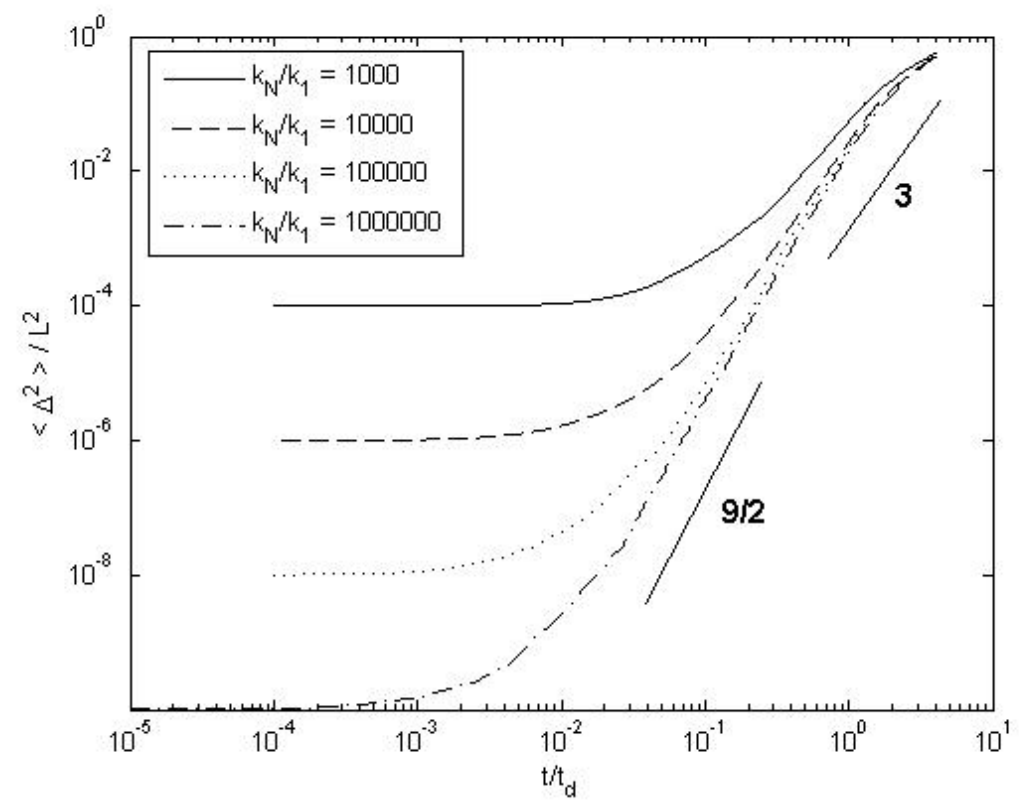

Fig. 2 Two particle separation as a function of time for different inertial subranges (cases $\mathrm{A}, \mathrm{F}, \mathrm{N}$ and $\mathrm{S}$ in Table 1). 


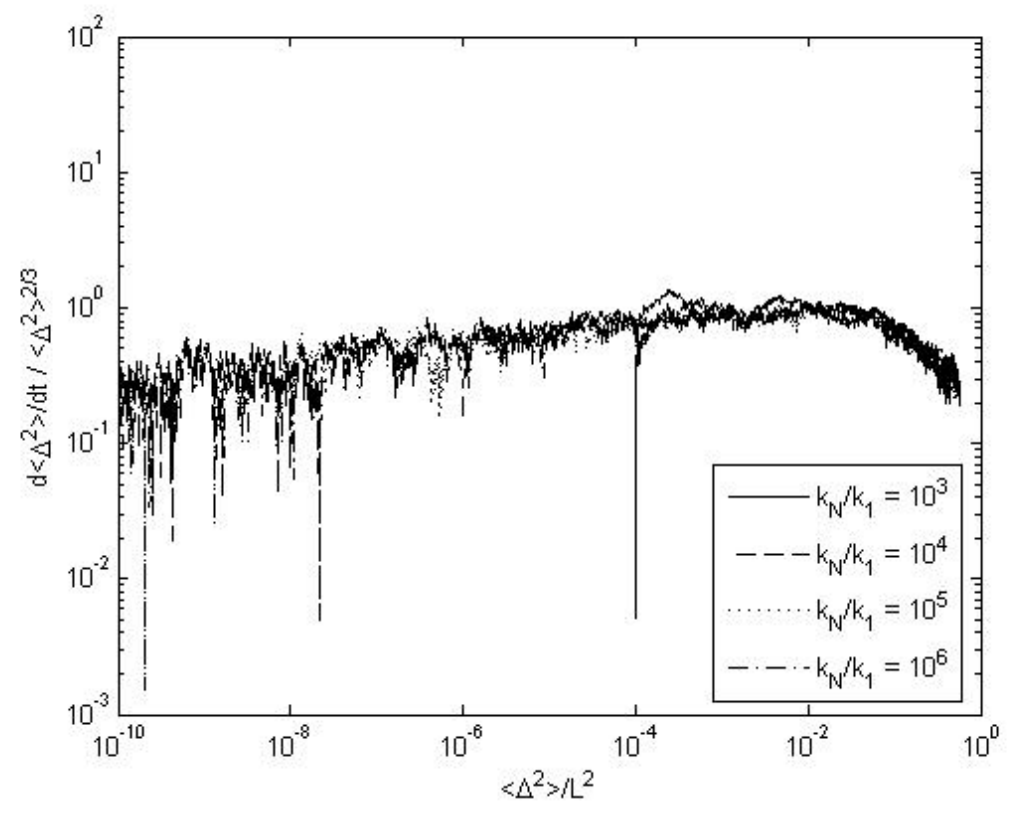

Fig. $3(\mathbf{d} / \mathbf{d t})<\Delta^{2}(\mathbf{t})>/<\Delta^{2}(\mathbf{t})>^{2 / 3}$ as a function of $\left\langle\Delta^{2}>/ \mathbf{L}^{2}, \mathbf{p}=5 / 3\right.$, same cases as in Fig. 2.

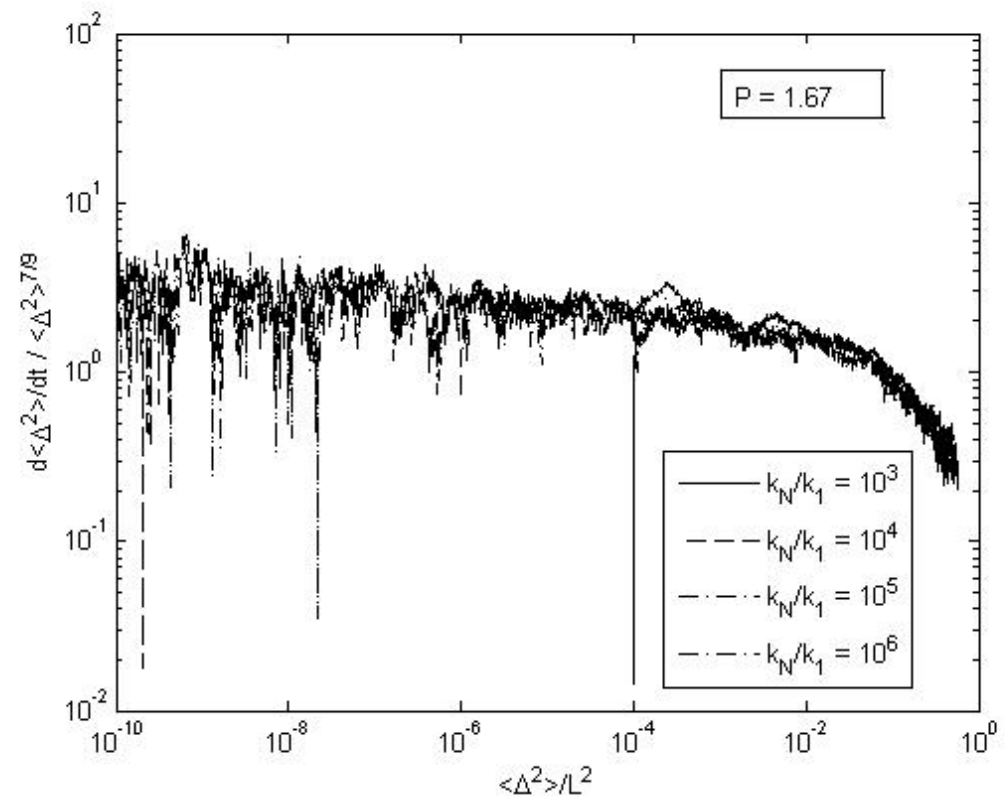

Fig. $4(\mathbf{d} / \mathbf{d t})<\Delta^{2}(\mathbf{t})>/<\Delta^{2}(\mathbf{t})>^{7 / 9}$ as a function of $\Delta^{2} / \mathbf{L}^{2}, \mathbf{p}=5 / 3$, same cases as in Fig. 2. 


\subsection{Sensitivity to the energy spectrum power law:}

Following $[2,16]$, Equation (12) can be written in a general form for a turbulence energy spectrum:

$\mathrm{E}(\mathrm{k}) \sim \mathrm{k}^{-\mathrm{p}}$

as follows:

$(\mathrm{d} / \mathrm{dt})<\Delta^{2}(\mathrm{t})>\sim<\Delta^{2}(\mathrm{t})>^{\mathrm{c}}$

which leads to

$<\Delta^{2}(\mathrm{t})>\sim \varepsilon \mathrm{t}^{(1 / 1-\mathrm{c})}$

With the following relations:

$1 /(1-c)=4 /(3-p)$

where $p$ is the energy spectrum exponent and varies as $1 \leq \mathrm{p} \leq 2$. When $\mathrm{E}(\mathrm{k}) \sim \mathrm{k}^{-5 / 3}$ we retrieve $\mathrm{c}=2 / 3$ and $\mathrm{t}^{3}$. Equation 16 is more general but still relies on Richardson's locality-inscale hypothesis.

In order to see the consistency of KS with this hypothesis and to have a better idea of the effect of increasing the inertial subrange on KS prediction of Richardson's law, we repeat previous results for different spectral power laws. We vary $p$ in Equation 15 from 1.27 to 1.97 and also vary the inertial range $\mathrm{k}_{\mathrm{N}} / \mathrm{k}_{1}$ (see the different cases reported in Table 1).

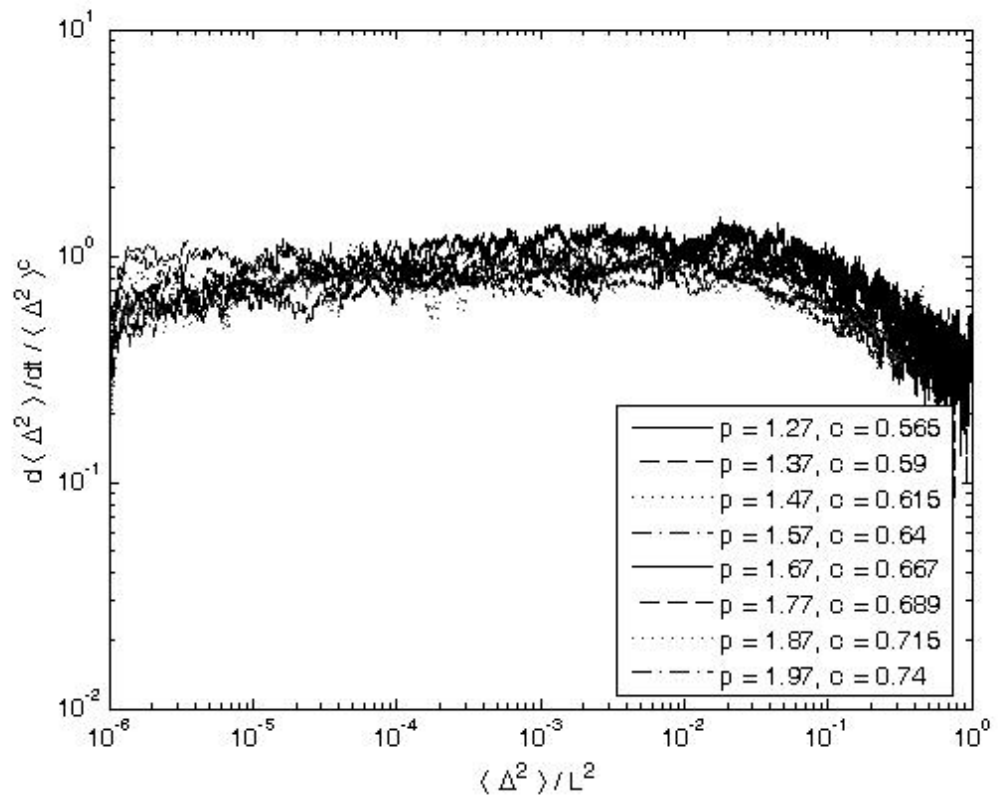

Fig. $5(\mathbf{d} / \mathbf{d t}))<\Delta^{2}(\mathbf{t})>/\left\langle\Delta^{2}(\mathbf{t})\right\rangle^{\mathrm{c}}$ as a function of $\left\langle\Delta^{2}>/ \mathbf{L}^{2}\right.$ for different energy spectrum power laws and $k_{N} / k_{1}=10^{4}$, (cases $B, C, D, E, F, K, L$ and $M$ in Table 1). 


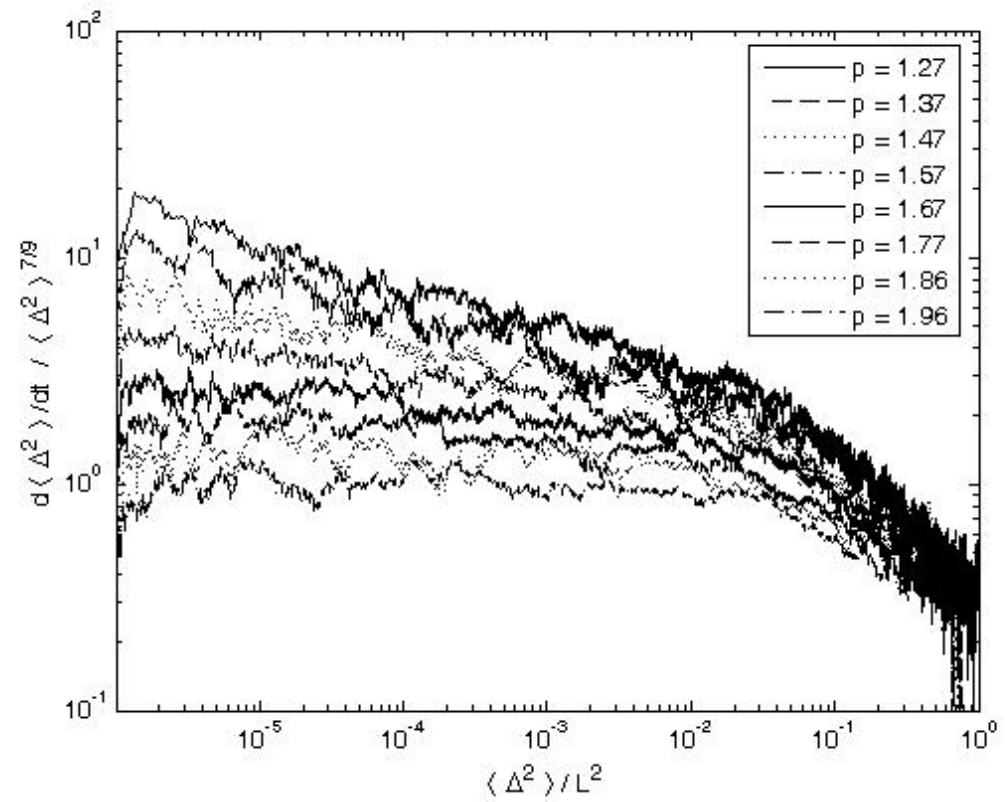

Fig. $6(\mathbf{d} / \mathbf{d t}))<\Delta^{2}(\mathbf{t})>/<\Delta^{2}(\mathbf{t})>^{7 / 9}$ as a function of $\left\langle\Delta^{2}>/ \mathbf{L}^{2}\right.$ for different energy spectrum power laws and $k_{N} / k_{1}=10^{4}$ same cases as in Fig. 5 .

Figure 5 shows that all the curves collapse showing a remarkable consistency of KS with Richardson's locality-in-scale hypothesis and $[2,16]$ 's prediction (16).

For comparison we plot $\left.\left\langle\Delta^{2}(\mathrm{t})\right\rangle /<\Delta^{2}(\mathrm{t})\right\rangle^{7 / 9}$ as a function of $\left\langle\Delta^{2}>/ \mathrm{L}^{2}\right.$ for the same cases in Figures 6. We can conclude that Equation (16) is verified, that is the locality-in-scale hypothesis is verified for inertial ranges $\mathrm{k}_{\mathrm{N}} / \mathrm{k}_{1}<10^{4}$ and spectral powers $1.27 \leq \mathrm{p} \leq 1.97$. We repeat the computations for a larger inertial range $\mathrm{k}_{\mathrm{N}} / \mathrm{k}_{1}=10^{6}$ in Figures 7 and 8 respectively.

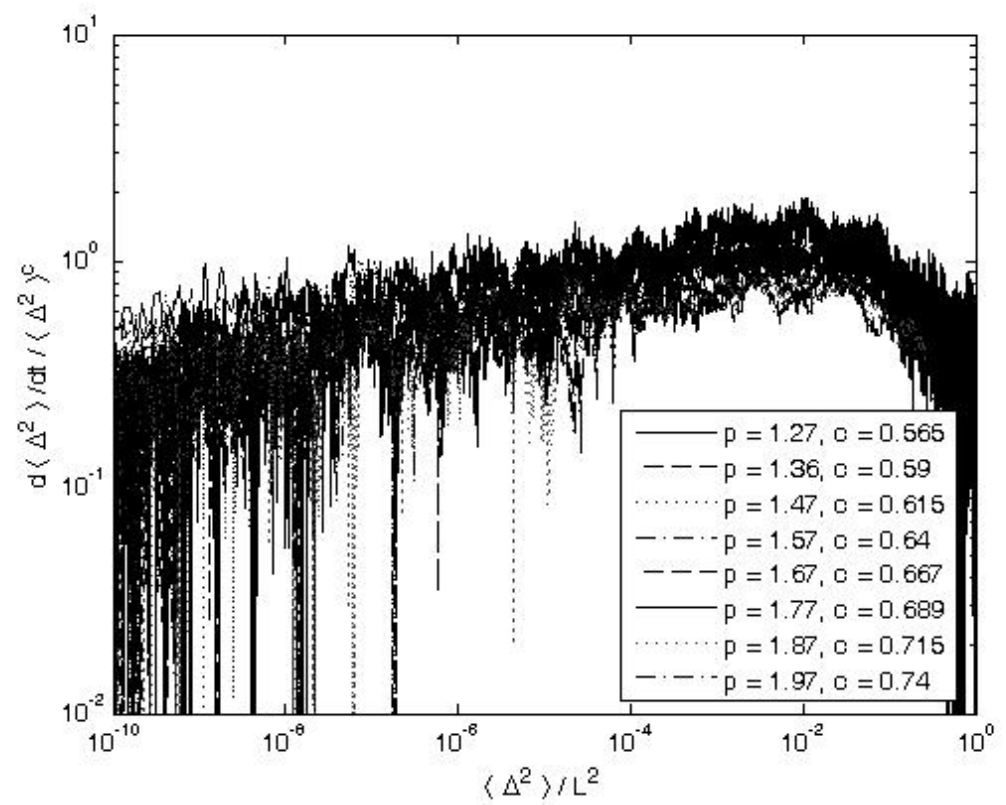

Fig. 7. (d/dt) ) $<\Delta^{2}(\mathbf{t})>/<\Delta^{2}(\mathbf{t})>^{\mathrm{c}}$ as a function of $\left\langle\Delta^{2}>/ \mathbf{L}^{2}, \mathbf{k}_{\mathrm{N}} / \mathbf{k}_{1}=10^{6}\right.$, (cases $\mathrm{O}, \mathrm{P}, \mathrm{Q}, \mathrm{R}, \mathrm{S}, \mathrm{V}, \mathrm{W}$ and $\mathrm{X}$ in Table 1 ). 


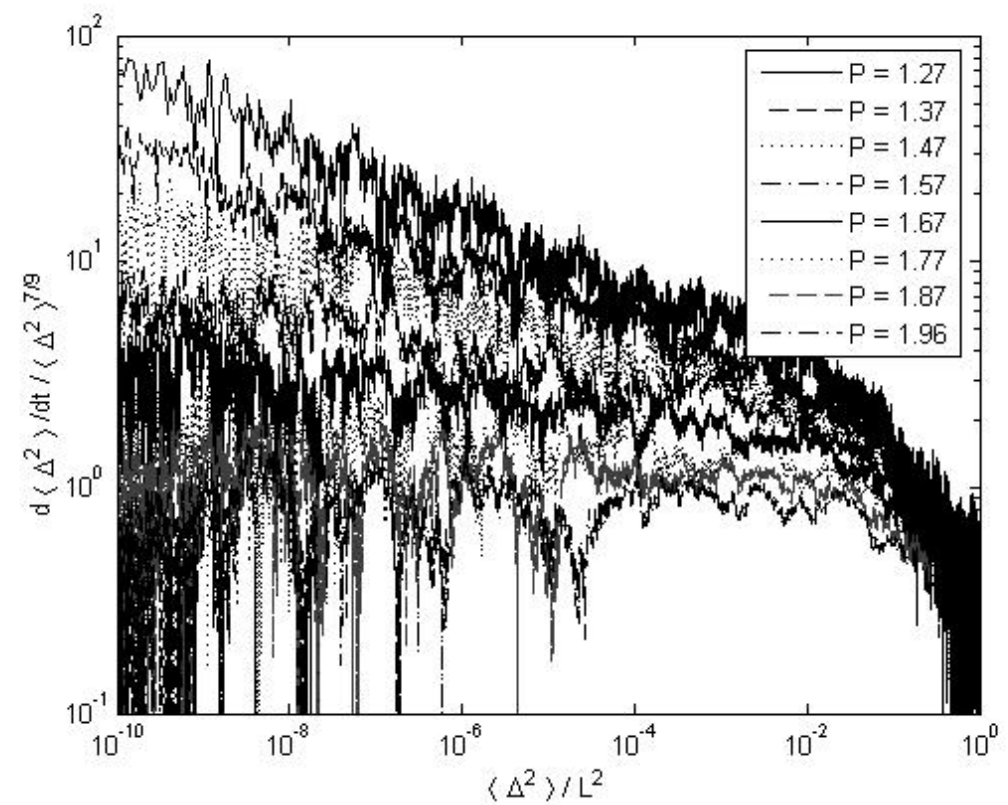

Fig. 8 (d/dt) ) $<\Delta^{2}(\mathbf{t})>/<\Delta^{2}(\mathbf{t})>^{7 / 9}$ as a function of $<\Delta^{2}>/ \mathbf{L}^{2}$ for different energy spectrum power laws and $k_{N} / k_{1}=10^{6}$, same cases as in Fig. 7 .

Figure 7 generalizes the conclusions made for $p=5 / 3$ that is $\mathrm{KS}$ is remarkably consistent with the locality assumption as all the curves collapse for the different values of $p$. When comparing Figures 7 and 8, we can also conclude that the locality-in-scale hypothesis and Equation 16 are verified for $1.27 \leq \mathrm{p} \leq 1.97$ and $\Delta / \mathrm{L} \geq 100$. Therefore KS difficulty to match Richardson's prediction is again localized to small scales $\left(\Delta / \mathrm{L} \leq 10^{-2}\right)$ and there is no effect of the spectral power law $\mathrm{p}$ on this range.

This questions the statement that KS cannot reproduce well Richardson's $\mathrm{t}^{3}$ because it does not accurately model the sweeping of small eddies by large eddies. The sweeping problem should be different with different spectral laws but we cannot see any effect of $p$ in Figure 7 . Furthermore for all values of $\mathrm{p}$ the problem is with small separations not with large separations which reproduce well $[2,16]$ 's prediction.

\subsection{Effect of varying the unsteadiness parameter on the Validity of Richardson Regime}

It is worth remembering that sweeping mechanisms have been proposed for $\mathrm{KS}$, the most popular is the term $\omega_{\mathrm{n}}$ defined in Equation (11). In all the cases we studied before, the unsteadiness parameter $\lambda$ was fixed to 0 as there is no conclusive results from previous researches showing it has any significant effect in three-dimensional KS.

In order to show if this parameter can have an effect on KS prediction of Richardson law, we repeat our results for $p=5 / 3$ for $0 \leq \lambda \leq 1$. Figure 9 corresponds to $k_{N} / k_{1}=10^{4}$ and Figure 10 to $\mathrm{k}_{\mathrm{N}} / \mathrm{k}_{1}=10^{6}$. 
From Figures 9 and 10, it can be noticed that $\lambda$ has no effect on the prediction of the diffusivity scaling. Even if the unsteadiness modeling, Equation (11), can be improved and if the sweeping was the main reason, why our KS cannot match Richardson's predictions at small separations we should see some alteration in the diffusivity when varying $\lambda$. However, the curves in Figures 9 and 10 collapse irrespective of the value of $\lambda$.

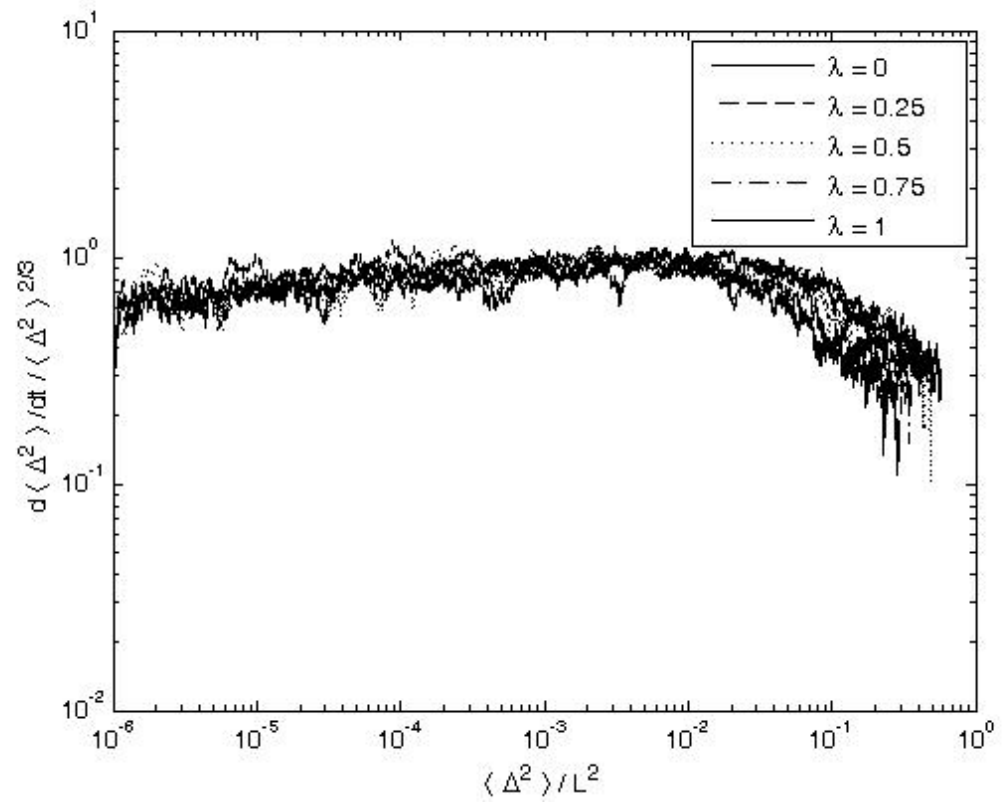

Fig. 9 Effect of the unsteadiness parameter on the normalized diffusivity with respect to Richardson's law as a function of the two-particle separations, $p=5 / 3$ and $k_{N} / k_{1}=10^{4}$, (cases F, G, H, I and $J$ in Table 1$)$.

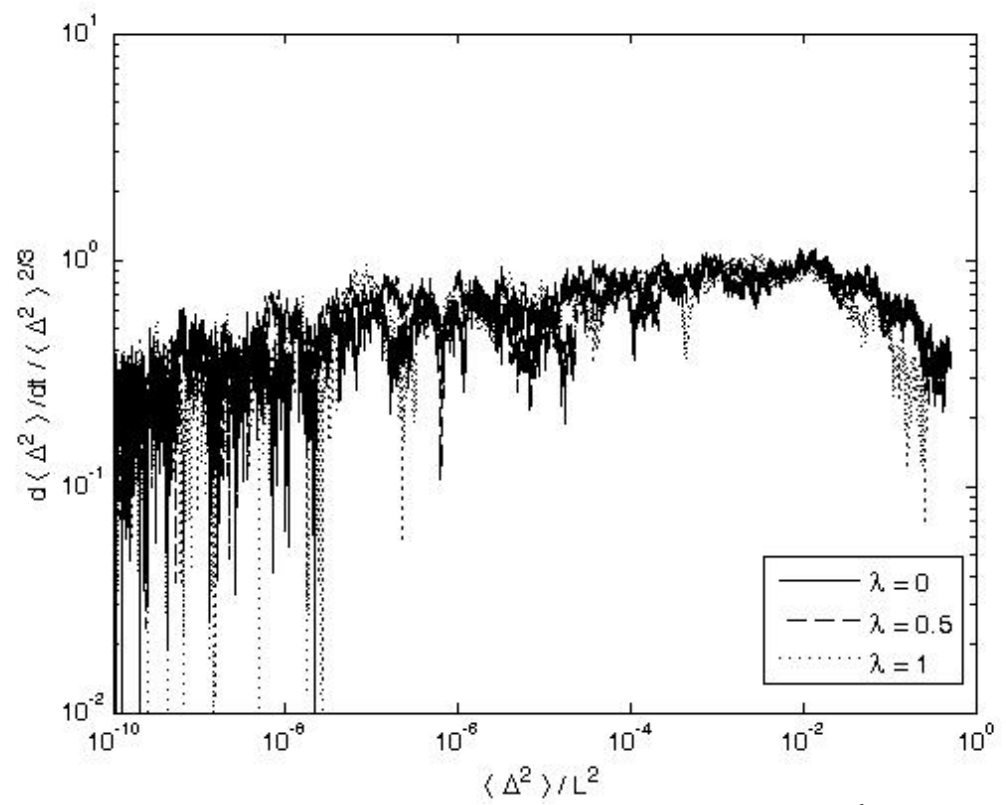

Fig. 10 Same as Fig. 9 but for $k_{N} / k_{1}=10^{6}$. 


\section{Conclusion}

The problem remains our KS prediction departs from [16]'s prediction at small scales for large inertial ranges. However, our results are still close to the theoretical prediction and it would be fairer to conclude that KS predictions are not as good at small separation as at large separation. For small inertial subrange, KS is efficient and the significance of the sweeping can be ignored, as a result we limit the KS agreement with the Richardson scaling law $\mathrm{t}^{3}$ for inertial subranges $\mathrm{k}_{\mathrm{N}} / \mathrm{k}_{1} \leq 10^{4}$. Above this value, the sweeping effect of the small scales by the large scales must be taken in considerations where KS failed to reproduces this power law in that range as obtained in [13].

\section{References}

[1] Richardson, L. F., "Atmospheric diffusion on a distance-neighbour graph", Proc. R. Soc. A 10, 523, 1926.

[2] Fung, J. C. H. and Vassilicos, J. C., "Two-particle dispersion in turbulent like flow", Physical Review E 57, 1677, 1998.

[3] Obukhov, A. M., "On the distribution of energy in the spectrum of turbulent flow ", Bull. Acad. Sci. U.S.S.R, Geog. \& Geophys., Moscow 5, 453, 1941.

[4] Batchelor, G. K., "The application of the similarity theory of turbulence to atmospheric dispersion “, Quart. J. R. Met. Soc. 76, 133, 1950.

[5] Jullien, M.C., Paret, J. and Tabeling, P., "Richardson pair dispersion in two-dimensional turbulence", Physical Review Letters 82, 2872, 1999.

[6] Yeung, P. K., "Direct numerical simulation of two-particle relative diffusion in isotropic turbulence“", Physics of Fluids 6, 3416, 1994.

[7] Malik, N. A. and Vassilicos, J. C., "A Lagrangian model for turbulent dispersion with turbulent-like flow structure: comparison with DNS for two-particle statistics", Physics of Fluids 11, 1572, 1999.

[8] Davila, J. and Vassilicos, J. C., "Richardson pair diffusion and the stagnation point structure of turbulence “, Physical Review Letters 91, 14501, 2003.

[9] Nicolleau, F. and Yu, G., "Two-particle diffusion and locality assumption", Physics of Fluids 16, 2309, 2004.

[10] Thomson, D. J. and Devenish, B. J., "Particle pair separation in kinematic simulations", J. Fluid Mech. 526, 277, 2005.

[11] Nicolleau, F. and Vassilicos, J. C., "Turbulent Pair Diffusion”, Physical Review Letters 90, 024503, 2003.

[12] Osborne, D. R., Vassilicos, J. C., Sung, K. and Haigh, J. D., "Fundamentals of pair diffusion in kinematic simulations of turbulence", Physical Review E 74, 036309, 2006.

[13] Devenish, B. J. and Thomson, D. J., "The effect of time-step size on particle pair separation in kinematic simulations", Physical Review E, Accepted, 2009.

[14] Nicolleau, F. and ElMaihy, A., "Study of the effect of the Reynolds number on threeand four-particle diffusion in three-dimensional turbulence using Kinematic Simulation", Physical Review E 74, 046302, 2006.

[15] Fung, J. C. H., Hunt, J. C. R., Malik, N. A. and Perkins, R. J., "Kinematic Simulation of homogeneous turbulence by unsteady random Fourier modes", J. Fluid Mech. 236, 281, 1992.

[16] Morel, P. and Larcheveque, M., "Relative Dispersion of Constant-Level Balloons in the 20mb General Circulation”, J. Atm. Sc. 31, 2189, 1974. 